\title{
Influência do tamanho do voxel na qualidade de imagem tomográfica: revisão de literatura
}

\author{
Influence of voxel size on the quality of tomography image: \\ literature review
}

\section{Resumo}

Objetivo: a menor unidade em espessura na imagem tomográfica é o voxel. Este foi definido antes da aquisição da imagem e pode ser alterado em busca de imagens tomográficas de qualidade. Diante disso, o objetivo deste artigo foi realizar uma revisão de literatura sobre a influência do tamanho do voxel na qualidade das imagens tomográficas. Revisão de literatura: a qualidade da imagem digital afeta a capacidade de identificar e delinear estruturas importantes para o diagnóstico. A imagem que apresenta uma qualidade adequada fornece informações suficientes para que o diagnóstico seja elaborado com um grau aceitável de segurança e confiabilidade. Dentro de outros parâmetros, o tamanho do voxel determinado na análise das imagens tomográficas tem sido um questionamento atual, com o objetivo de definir o melhor contraste e nitidez para identificação de estruturas anatômicas e processos patológicos. Considerações finais: a imagem adequada ao diagnóstico deve proporcionar sempre a maior segurança à sua interpretação. Assim, deve-se sempre utilizar o tamanho do voxel no protocolo de aquisição mais adequado ao fim específico do diagnóstico.

Palavras-chave: Tomografia. Controle de qualidade. Diagnóstico por imagem.

\section{Introdução}

A definição de qualidade de imagem para tomografia computadorizada (TC) foi descrita como a capacidade de visibilidade para diagnóstico de estruturas importantes na imagem de TC ou a visualização de estruturas anatômicas relevantes e como a capacidade de detectar achados patológicos ${ }^{1,2}$.

Imagens de radiodiagnóstico devem prover informações suficientes para permitir que médicos e cirurgiões-dentistas tomem decisões com um grau razoável de certeza e propiciar dose de radiação mínima para o paciente, respeitando-se o princípio ALARA (as low as reasonably achievable) ${ }^{3}$.

A impressão subjetiva de qualidade de imagem depende de diversos fatores, como nitidez de contorno, nível de ruído e contraste entre as estruturas. Outro fator que contribui para a qualidade de imagem é a presença ou a ausência de artefatos, levando em consideração sua intensidade e sua influência na detecção de patologias e estruturas anatômicas relevantes ${ }^{2}$. 
Dispositivos e processos de aquisição podem afetar a qualidade da imagem. Durante a aquisição da imagem, o operador necessita saber as variáveis do protocolo: o campo de visão (FOV - field of view), o tamanho do voxel, o tempo de varredura, os parâmetros de $\mathrm{mA}$, de $\mathrm{kVp}$ e, também, a imobilização do paciente ${ }^{2,4}$.

A menor unidade em espessura na imagem tomográfica é o voxel, o qual é definido antes da aquisição da imagem e pode ser alterado, em busca de imagens tomográficas que proporcionem uma melhor nitidez e contraste de estruturas anatômicas e processos patológicos. Diante disso, o objetivo deste artigo foi realizar uma revisão de literatura sobre a influência do tamanho do voxel na qualidade de imagem das TCs.

\section{Revisão de literatura}

A imagem tomográfica é uma imagem matricial em que o arranjo das linhas e colunas forma os elementos de imagem denominados individualmente de pixel, a área resultante da interseção das linhas com as colunas. Os pixels são os menores componentes de imagem e têm dimensões regulares específicas. Na imagem radiográfica digital, a cor do pixel é cinza, de tal forma que as intensidades que variam do preto ao branco são distribuídas em um espectro da escala de tons cinza com $2^{8}$ (256) tonalidades em imagens de 8 bits ou $2^{12}$ (4.096) tonalidades em imagens de 12 bits. A nitidez ou resolução da imagem está, em última instância, relacionada ao tamanho do pixel e ao número de tonalidades de cinza ${ }^{5}$.

Para que a imagem de tomografia possa ser reconstruída de modo a mostrar as estruturas em sua forma real, são necessárias múltiplas tomadas de dados em diferentes ângulos de projeção. A partir dos dados obtidos em cada leitura, o computador interpreta o grau de densidade dos diferentes tecidos, atribuindo a cada um o valor correspondente de uma escala de cinzas. O resultado final é apresentado pelos pixels que formam a imagem tomográfica ${ }^{6,7}$.

A espessura do corte forma a terceira dimensão e está relacionada à profundidade do corte. $\mathrm{O}$ volume formado pelo pixel e pela profundidade do corte é conhecido por voxel. Um exame tomográfico contém em torno de 100 milhões de voxels, representando cada um deles um grau de absorção dos raios $\mathrm{X}$ e um valor específico de cinza em imagens bidimensionais no filme ou no monitor do computador?.

Erros associados a dados volumétricos estão relacionados, fundamentalmente, ao tamanho do voxel e à qualidade dos dados (8 bits x 12 bits), pois 0 valor da intensidade atribuído a cada voxel depende da média de intensidade ou da atenuação do feixe pelo material contido em seu volume. Com um tamanho pequeno de voxel, ocorre uma atribuição mais acurada de um valor de atenuação. Da mesma forma, quando existem mais possibilidades de valores de intensidade, como em imagens de 8 bits ou 12 bits, há uma possibilidade progressivamente maior de escolha para o valor real da intensidade ${ }^{5}$.

O tamanho do voxel determina o nível de detalhes que uma imagem proporciona, ou seja, sua resolução. Em TC médica, os voxels são, via de regra, anisotrópicos (cubos irregulares, onde a maior dimensão do voxel é a espessura axial). Embora a superfície do voxel em TC possa ser tão pequena quanto $0,625 \mathrm{~mm}$, sua profundidade é, geralmente, na ordem de $1 \mathrm{~mm}$ a $2 \mathrm{~mm}$. Já as unidades de tomografia computadorizada de feixe cônico (TCFC) possuem voxels isotrópicos, isto é, iguais em todas as três dimensões ${ }^{2,8-10}$.

Quanto menor o voxel na aquisição da imagem e mais longo o tempo de varredura, melhores serão a resolução e os detalhes. No entanto, um tamanho menor de voxel está atrelado a um tempo de varredura mais longo, o que proporciona algumas desvantagens, como maiores possibilidades de movimentação do paciente durante o exame, doses mais elevadas de radiação e tempo de reconstrução mais longo ${ }^{11}$.

A alta resolução não significa, necessariamente, alta qualidade de imagem. Tamanho do voxel, $\mathrm{mA}$ e qualidade de imagem estão intimamente relacionados. A mA controla o número de fótons liberados pelo tubo de raios $\mathrm{X}$ e, posteriormente, o número de fótons que atingem os voxels. Se o número de fótons liberado for mantido constante ( $\mathrm{mA} \mathrm{e}$ tempo de aquisição constantes) e o tamanho do voxel do detector for alterado, o número de fótons que atinge $o$ voxel também mudará. Nesse caso, voxels menores são atingidos por uma menor quantidade de fótons, e voxels maiores são atingidos por uma maior quantidade de fótons. Quanto mais fótons atingem cada voxel, mais informações cada um deles gera, contribuindo, assim, para uma diferença na qualidade de imagem, pois o ruído diminui com a elevação do tamanho do voxel. Assim, quando o tamanho do voxel é diminuído, mais fótons são necessários para aumentar a contagem de fótons por voxel, para que a qualidade da imagem permaneça a mesma ${ }^{1,12-15}$.

O tamanho do voxel, de maneira independente, não influencia na dose de radiação, ou seja, quando os fatores de exposição (tempo de exposição, $\mathrm{kVp}$ e $\mathrm{mA}$ ) são mantidos, a simples alteração do tamanho do voxel não influencia de maneira significativa a dose de radiação. No entanto, os protocolos atrelam o uso de voxels menores a tempos de exposição e mA maiores, o que, invariavelmente, provoca uma melhor resolução da imagem e o aumento da dose de radiação. Isso ocorre quando os protocolos são totalmente preestabelecidos pelo fabricante de alguns aparelhos de $\mathrm{TCFC}^{11,16,17}$.

Para comparar a qualidade de imagem de dois diferentes aparelhos de TCFC, o Siremobil Iso-C3D (Siemens Medical Solutions, Erlangen Alemanha) e 
o NewTom 9000 (NIM srl, Verona, Itália), foram adquiridas imagens de um crânio seco humano. Diferentes estruturas anatômicas foram analisadas por dez observadores nas reconstruções multiplanares. Em relação à qualidade de imagem, não houve diferença estatisticamente significativa entre os dois aparelhos, tendo apenas o canal mandibular e o osso trabecular, na reconstrução ortogonal, apresentado uma qualidade de imagem maior no TCFC NewTom 9000, para o qual, no protoloco de aquisição, o voxel foi menor que para o Siremobil Iso-C3D ${ }^{18}$.

Um estudo analisou a capacidade de diagnóstico das imagens por TCFC i-CAT, com diferentes tamanhos de voxel, para identificação de reabsorções radiculares externas simuladas com a utilização de broca. Os dentes incisivos inferiores selecionados não foram seccionados, mas as suas raízes foram distribuídas em terços cervical, médio e apical, com quatro possibilidades para cada terço: cavidade pequena, média, grande e sem cavidade. Foram adquiridas imagens de 59 dentes inseridos em uma base de cera para simular os efeitos do tecido mole, em três protocolos nos quais a variação foi o tamanho do voxel $(0,2$ $\mathrm{mm}, 0,3 \mathrm{~mm}, 0,4 \mathrm{~mm}$ ). Um examinador calibrado e sem conhecimento dos protocolos avaliou as imagens nos planos axial, coronal e sagital no programa i-CAT View ${ }^{\circledR}$ (Imaging Sciences International, Inc.), e não foram encontradas diferenças estatisticamente significativas entre os tamanhos do voxel, plano, tamanho da cavidade e terço radicular ${ }^{10}$.

A acurácia de mensurações lineares realizadas na mandíbula por meio de imagens de TCFC i-CAT adquiridas com diferentes tamanhos de voxel foi avaliada com o objetivo de estabelecer o melhor protocolo a ser utilizado para realização de mensurações lineares. Oito mandíbulas secas (com sítios estabelecidos) foram submetidas a exames de TCFC, a partir de quatro protocolos, com $\mathrm{kVp}$ constante de 120 e com os seguintes tamanhos de voxel, tempo para cada aquisição e $\mathrm{mA}$, respectivamente: $\mathrm{p} 1$ - voxel de 0,2 mm, 40s e 46,72 mA; p2 - voxel de $0,25 \mathrm{~mm}, 40 \mathrm{~s}$ e $46,72 \mathrm{~mA}$; $\mathrm{p} 3$ - voxel de $0,3 \mathrm{~mm}, 20 \mathrm{~s}$ e 23,87 mA; p4 - voxel de 0,4 mm, 20s e $23,87 \mathrm{~mA}$. A acurácia das mensurações verticais e horizontais em TCFC (i-CAT) obtidas com diferentes tamanhos de voxel mostrou-se comparável às mensurações realizadas na mandíbula seca ${ }^{11}$.

Com o aparelho de TCFC NewTom $3 G^{\circledR}$, foram adquiridas imagens tomográficas de 63 cabeças de ovelhas sem e com fraturas condilares criadas, com vistas a determinar a precisão de diagnóstico de diferentes espessuras de cortes nas reconstruções de TCFC. Sete observadores avaliaram as reconstruções multiplanares com espessuras de corte de $0,2 \mathrm{~mm}, 1 \mathrm{~mm}, 2 \mathrm{~mm}$ e $3 \mathrm{~mm}$ e concluíram que a TCFC foi precisa em detectar todos os tipos de fratura nas espessuras de corte de $0,2 \mathrm{~mm}$ e $1 \mathrm{~mm}$; porém, os cortes de $2 \mathrm{~mm}$ e $3 \mathrm{~mm}$ não foram adequados para detectar fissuras completas e cominutivas da cabeça da mandíbula ${ }^{19}$.
A precisão das mensurações lineares e a detecção do canal mandibular em imagens tomográficas de feixe cônico foram avaliadas em diferentes tamanhos de voxel. Para isso, doze hemimandíbulas humanas secas foram digitalizadas em tamanhos voxel de $0,2 \mathrm{~mm} ; 0,3 \mathrm{~mm} ; 0,4 \mathrm{~mm}$, tendo sido geradas 108 imagens ortogonais de TCFC i-CAT. Dois radiologistas realizaram 648 mensurações lineares e avaliaram a visibilidade do canal mandibular. Os autores concluíram que as reconstruções ortogonais foram adequadas para as mensurações lineares na região posterior da mandíbula e proporcionaram visualização adequada do canal mandibular nos três tamanhos de voxel utilizados ${ }^{20}$.

\section{Considerações finais}

Os estudos demonstram que quanto menor o tamanho do voxel, maior a qualidade de imagem; porém, a redução do tamanho do voxel está quase sempre associada ao aumento de $\mathrm{mA}$ e do tempo de exposição. Reduzir o tamanho do voxel sem elevar mA, característica de alguns novos equipamentos, seria, em tese, uma vantagem, embora alguns estudos tenham mostrado não haver diferença na qualidade de imagem para determinados fins, quando da utilização de voxels de tamanho maior, pois isso aumentaria a espessura da estrutura, facilitando a sua visualização.

A imagem adequada ao diagnóstico deve proporcionar sempre a maior segurança à sua interpretação. Assim, deve-se considerar, respeitando o princípio ALARA (as low as reasonably achievable), sempre o protocolo de aquisição mais adequado ao fim específico do diagnóstico.

\section{Abstract}

Objective: the smallest thickness unit in the tomographic image is the voxel. This is set before image acquisition and may be changed in the search of quality tomographic images. Thus, this article aimed to perform a literature review on the influence of voxel size on the quality of tomography images. Literature review: the quality of the digital image affects the ability to identify and outline structures which are important for diagnosis. The image presenting proper quality provides enough information for the development of a diagnosis with acceptable level of safety and reliability. Within other parameters, the voxel size determined in tomographic image analysis is currently undergoing questioning, aiming to define the best contrast and sharpness to identify anatomical structures and pathological processes. Final considerations: the image suitable for diagnosis should always provide the highest security for its interpretation. Thus, one should always use the voxel size at the acquisition protocol best suited for the specific purpose of diagnosis.

Keywords: Tomography. Quality control. Diagnostic imaging. 


\section{Referências}

1. Kwong JC, Palomo JM, Landers MA, Figueroa A, Hans MG. Image quality produced by different cone-beam computes tomography settings. Am J Orthod Dentofacial Orthop 2008; 133(2):317-27.

2. Mischkowski RA, Scherer P, Ritter L, Neugebauer J, Keeve $\mathrm{E}$, Zöller JE. Diagnostic quality of multiplanar reformations obtained with a newly developed cone beam device for maxillofacial imaging. Dentomaxillofac Radiol 2008; 37(1):1-9.

3. Martin CJ, Sharp PF, Suttom DG. Measurement of image quality in diagnostic radiology. Appl Radiat Isot 1999; 50(1):21-38.

4. Hatcher DC. Operational principles for cone-beam computed tomography. J Am Dent Assoc 2010; 141(3):3S-6S.

5. Stratemann SA, Huang JC, Maki K, Miller AJ, Hatcher DC. Comparison of cone beam computed tomography imaging with physical measures. Dentomaxillofoc Radiol 2008; 37(2):80-93.

6. Pinsky HM, Dyda S, Pinsky RW, Misch KA, Sarment DP. Accuracy of three-dimensional measurements using conebeam CT. Dentomaxillofoc Radiol 2006; 35(6):410-6.

7. Patel S, Dawood A, Ford TP, Whaites E. The potential applications of cone beam computed tomography in the management of endodontic problems. Int Endod J 2007; 40(10):818-30

8. Scarfe WC, Farman AG, Sukovic P. Clinical applications of cone-beam computed tomography in dental practice. J Can Dent Assoc 2006; 72(1):75-80.

9. Connor SE, Arscott T, Berry J, Greene L, O'Gorman R. Precision and accuracy of low-dose CT protocols in the evaluation of skull landmarks. Dentomaxillofac Radiol 2007; 36(5):270-6.

10. Liedke GS, da Silveira HE, da Silveira HL, Dutra V, de Figueiredo JA. Influence of voxel size in the diagnostic ability of cone beam tomography to evaluate simulated external root resorption. J Endod 2009; 35(2):233-5.

11. Torres MGG, Campos PSF, Pena N, Ribeiro M, Navarro M, Crusoé-Rebello I. Avaliação de doses referenciais obtidas com exames de tomografia computadorizada de feixe cônico adquiridos com diferentes tamanhos de voxel. Dental Press J Orthod 2010; 15(5):42-3.

12. Araki K, Maki K, Seki K, Sakamaki K, Harata Y, Sakaino $\mathrm{R}$, et al. Characteristics of a newly developed dentomaxillofacial X-ray cone beam CT scanner (CB MercuRaye): system configuration and physicalproperties. Dentomaxillofac Radiol 2004; 33(1):51-9.

13. Tanimoto H, Arai Y. The effect of voxel size on image reconstruction in cone-beam computed tomography. Oral Radiol 2009; 25(2):149-53.

14. Hassan B, Couto Souza P, Jacobs R, de Azambuja Berti S, van der Stelt $\mathrm{P}$. Influence of scanning and reconstruction parameters on quality of three-dimensional surface models of the dental arches from cone beam computed tomography. Clin Oral Investig 2010; 14(3):303-10.

15. Moshfeghi M, Tavakoli MA, Hosseini ET, Hosseini AT, Hosseini IT. Analysis of linear measurement accuracy obtained by cone beam computed tomography (CBCT-NewTom VG). Dent Res J 2012; 9(Suppl 1):S57-62.

16. Ludlow JB, Davies-Ludlow LE, Brooks SL, Howerton WB. Dosimetry of 3 CBCT devices for oral and maxillofacial radi- ology: CB Mercuray NewTom 3G and i-Cat. Dentomaxillofac Radiol 2006; 35(4):219-26.

17. Maret D, Telmon N, Peters OA, Lepage B, Treil J, Inglèse JM, et al. Effect of voxel size on the accuracy of 3D reconstructions with cone beam CT. Dentomaxillofac Radiol 2012; 41(8):649-55.

18. Schulze D, Heiland M, Blake F, Rother U, Schmelzle R. Evaluation of quality of reformatted images from two conebeam computed tomographic systems. J Cranio-Maxillofac Surg 2005; 33(1):19-33.

19. Sirin Y, Guven K, Horasan S, Sencan S, Bakir B, Barut O, et al. The influence of secondary reconstruction slice thickness on NewTom 3G cone beam computed tomography-based radiological interpretation of sheep mandibular condyle fractures. Oral Surg Oral Med Oral Pathol Oral Radiol Endod 2010; 110(5):638-47.

20. Waltrick KB, Nunes de Abreu Jr MJ, Corrêa M, Zastrow MD, Dutra VD. Accuracy of linear measurements and visibility of the mandibular canal of cone-beam computed tomography images with different voxel sizes: an in vitro study. J Periodontol 2013; 84(1):68-77.

\section{Endereço para correspondência:}

Cinthia Coelho Simões

Rua Albatroz, 213, apt. 701플 Imbui

41.720-420 Salvador-BA

Fones: 71-8833-3305/ 3234-0061

E-mail: cincoelhounime@gmail.com

Recebido: 18/08/2013. Aceito: 26/11/2013. 